\title{
Revealing the Toxicity of Chlorate Through the Analysis of Molecular Interaction using Viscosity Measurements and Apparent Molar Volumes
}

\author{
MEENAKSHI V. RATHI \\ Department of Chemistry,RNC Arts, JDB Commerce, NSC Science College, \\ Nashik Road, Nashik.(Maharashtra) India. \\ *Corresponding author E-mail: meenakship2@gmail.com
}

http://dx.doi.org/10.13005/ojc/370610

(Received: October 20, 2021; Accepted: December 02, 2021)

\begin{abstract}
While chlorate has the ability to induce flowering in longan, it also has adverse impacts on the crop. Revealing the toxicity of chlorate in the environment is more than just about the environment and about human health, as well. Because of the large introduction of this chemical into the environment from the paper processing industry, there is indeed a lot of concern about its toxicity. Chlorate toxicology in the longan plant has been thoroughly investigated in solutions using viscosities and apparent molar volumes. The hydration of molecules and volume changes are involved in various chemical and biological processes in plant tissues, and their complete understanding demands a good idea for volumetric and viscometric study. It offers good data acquisition techniques for solute, solvent and solvent-solvent interactions. Multi-component systems containing $\mathrm{KClO}_{3}+$ water + ionic solid (ionic solids $=\mathrm{KCl}, \mathrm{KNO}_{3}, \mathrm{NH}_{4} \mathrm{NO}_{3}$ and $\mathrm{KH}_{2} \mathrm{PO}_{4}$, are currently being worked out to study the dependence of transport properties of potassium chlorate in aqueous electrolyte solutions, with concentrations and temperature of solutions. The assessed kd values are used to predict whether the solvolysis of $\mathrm{KClO}_{3}$ in the presence of other electrolytes is a quick or slow process.
\end{abstract}

Keywords: Toxicity, $\mathrm{KClO}_{3}$, Volumetric and Viscometric properties, Diffusion controlled Reaction Rate Constant $\mathrm{k}_{\mathrm{d}}$.

\section{INTRODUCTION}

Lychees and longans are close family members. longans and lychees are comparable in nutritional composition. Both are minimal in calories and carbohydrates and are nil in fat. The antioxidants and fibres are abundant in longan and lychee similarly are great source of vitamin C.



Fig. 1. Lychee Fruit

This is an Open Access article licensed under a Creative Commons license: Attribution 4.0 International (CC- BY). Published by Oriental Scientific Publishing Company @ 2018 


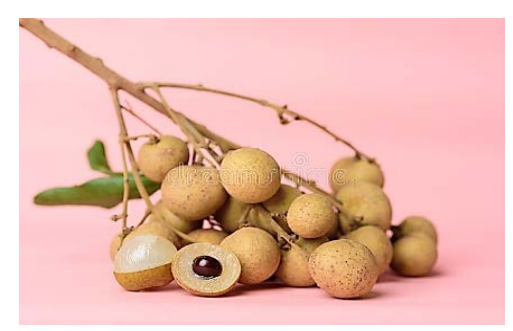

Fig. 2. Longan Fruit

Longan (Dimocarpus longan), a subtropical fruit plant belonging to the Sapindaceae family, is ideally suited to tropical regions with distinct wet/ dry seasons as well as subtropical locales with a cool, nonfreezing fall/winter period. In India, it is one of the minor fruits. There is currently insufficient documentation on longan production in India to analyze the real scenario. The longan is an important crop in Southeast Asia economically. Although leaves and flowers are sold in markets, they are not used in traditional medicine. The dried flowers are shipped to Malaysia to be used as medicine.

Off-season flowering and fruit production is a desirable economic and scientific goal for many subtropical fruit plants. The discovery of potassium chlorate to induce off-season flowering in longan, as well as off-season longan production methods in Thailand, including soil drench (Manochai S. W.-a., 1999) ((Sritontip C. Y., 1999)), foliar spray ((Sritontip C. Y., 1999)), and truck or stem injection ((Viriya-alongkorn, 1999)).

Though chlorate is able to precisely induce flowering in longan, it also causes toxic effects on the crop. $\mathrm{KClO}_{3}$ serves as a herbicide or a defoliant on many other plants. (Nahar, 2010) (Manochai P. J., August 2008)

While chlorate is able to specifically induce flowering in longan, it also causes toxic effects on the crop. (Sritontip C. Y., 2003). Great concern is paid upon the toxicity of chlorate (Daniel, 1990) because of heavy introduction of this chemical into the environment from paper making industry. (Moore \& Calabrese, 1982). An increasing demand from end user industries, such as farming, explosives, laboratories and disinfectants is predicted to contribute significantly to the growth of the potassium chlorate market in the forecast time frame. The explosives based on potassium chlorate are more effective than ordinary powder. The production of fireworks and matching bands also involves extensively utilised potassium chlorate. In smoke compositions such as smoke grenades it is utilised as a component. In laboratories and educational facilities, potassium chlorate is used to generate oxygen, other than explosives. It is also utilised for the destruction of pests as a pesticide in agriculture. (https://www.businesswire.com/portal/site/home/ my-business-wire/, 2020).

A significant amount of information is needed on the properties of transport, thermodynamic and solution to comprehend how the co-solvent or co-solute can change the nature and structure of the water. (Bahadur, 2019).

This work examines the interaction like ion-solvent, ion-ion and solvent-solvent of potassium chlorate with aqueous ionic salts, as both the solute and solvents are utilised for agricultural operations. In this study the chemical and the electrostatic effect due to dielectric constant of the co-solute which are already present in soil like $\mathrm{KCl}, \mathrm{KNO}_{3}$ and $\mathrm{NH}_{4} \mathrm{NO}_{3}$, $\mathrm{KH}_{2} \mathrm{PO}_{4}$ would play vital role in influencing solutesolvent interaction. (Ganjare, 2020). Viscosity data can be used to determine the diffusion controlled reaction rate constant $\mathrm{k}_{\mathrm{d}}$.

The current study used multi-component systems containing an ionic solid, water, and $\mathrm{KClO}_{3}$ (ionic solids $=\mathrm{KCl}, \mathrm{KNO}_{3}$, and $\mathrm{NH}_{4} \mathrm{NO}_{3}$, $\left.\mathrm{KH}_{2} \mathrm{PO}_{4}\right)$ ) to evaluate the relationship between the transport properties of $\mathrm{KClO}_{3}$ solutions in aqueous electrolyte solutions and solution concentrations and temperature.

The densities of aqueous solutions were measured using $15 \mathrm{~cm}^{3}$ double arm pycnometer in a transparent glass walled water bath. (Kharat, 2007) Triple distilled water was used to calibrate the pycnometer. At desired temperatures, the densities of $\mathrm{KClO}_{3}$ solutions in aqueous $1 \% \mathrm{KCl}, \mathrm{KNO}_{3}$ and $\mathrm{NHNO}_{3}, \mathrm{KH}_{2} \mathrm{PO}_{4}$, and pure water were determined using a bi-capillary pycnometer. An average of triplicate measurements was the final result and the density was calculated with an accuracy of $\pm 1.25 \times 10^{-4} \mathrm{~g} . \mathrm{cm}^{-3}$. The thermostat temperature is maintained at the appropriate temperatures using a demerstat with an accuracy of $\pm 0.1 \mathrm{~K}$.

Viscosities of all salt solutions of ten different concentrations in water were measured using Ubbelohde viscometer at temperatures of 298.15 , 
303.15, 308.15, and 313.15K. Measurements to monitor reproductibility of results have been repeated at least three times The overall precision of viscosity measurements was $\pm 2.0 \times 10^{-4} \mathrm{mPa}$.s. At 0.01 second intervals the flow time is accurately recorded.
All chemicals were taken from Sigma Aldrich, Germany, with more than $99 \%$ purity and was further desiccated over anhydrous $\mathrm{CaCl}_{2}$ before use. (Nanda, Nanda, \& Mohanty, 2012), The sample specification is given in Table 1a,b.

Table 1(a): Specification of Chemicals

\begin{tabular}{|c|c|c|c|c|c|c|}
\hline Chemicals & Source & Mass fraction Purity & Purification Method & CAS No. & Mol. Mass g. Mol-1 & Chemical Formula \\
\hline Potassium Chlorate & $\begin{array}{l}\text { Sigma } \\
\text { Aldrich }\end{array}$ & $\geq 99.0 \%$ & $\begin{array}{l}\text { desiccated over } \\
\text { anhydrous } \mathrm{CaCl}_{2}\end{array}$ & $4 / 9 / 3811$ & 122.55 & $\mathrm{KClO}_{3}$ \\
\hline Potassium Nitrate & $\begin{array}{l}\text { Sigma } \\
\text { Aldrich }\end{array}$ & $\begin{array}{c}\text { ACS reagent } \\
, \geq 99.0 \%\end{array}$ & Used as obtained & $7757-79-1$ & 101.1 & $\mathrm{KNO}_{3}$ \\
\hline Potassium Chloride & $\begin{array}{l}\text { Sigma } \\
\text { Aldrich }\end{array}$ & $\geq 99.0 \%$ & Used as obtained & $7447-40-7$ & 74.55 & $\mathrm{KCl}$ \\
\hline Ammonium Nitrate & $\begin{array}{l}\text { Sigma } \\
\text { Aldrich }\end{array}$ & $\begin{array}{l}\text { ACS reagent, } \\
\geq 98 \%\end{array}$ & Used as obtained & $6484-52-2$ & 80.04 & $\mathrm{NH}_{4} \mathrm{NO}_{3}$ \\
\hline $\begin{array}{l}\text { Potassium phosphate } \\
\text { monobasic }\end{array}$ & $\begin{array}{l}\text { Sigma } \\
\text { Aldrich }\end{array}$ & $\geq 98.0 \%$ & Used as obtained & 7778-77-0 & 136.1 & $\mathrm{KH}_{2} \mathrm{PO}_{4}$ \\
\hline
\end{tabular}

It is known the properties like density, viscosity, etc., are influenced by the presence of oxygen atom in a molecule. The presence of such groups increases the hydrophilicity and electrostriction between water and solute particles. (Nanda, Nanda, \& Dalai, 2021).

\section{Data Evaluation}

The apparent molar volumes $\varnothing_{v}$, were obtained from the density results using the following equation (Cui, et al., 2020) (Langore, Nikumbh, Patil, \& Gaikwad, 2019).

$\emptyset_{V}=\frac{1000\left(\rho_{0}-\rho\right)}{C \rho_{0}}+\frac{M}{\rho}$

Where $\mathrm{M}$ is the molar mass of the $\mathrm{KClO}_{3}$ in $\mathrm{g} \mathrm{mol}^{-1}, \mathrm{C}$ is the concentration (mol. $\left.\mathrm{L}^{-1}\right), \rho$ and $\rho_{o}$ and the densities of the solution and the solvent, respectively in $\mathrm{g} \mathrm{cm}^{-3}$

The apparent molar volumes $\left(\varnothing_{v}\right)$ were plotted against the square root of concentration $\left(\mathrm{C}^{1 / 2}\right)$ in accordance with the Masson's equation. Partial molar volumes at infinite dilution were obtained by least-squares fitting to the equation: (Caro, 2020).

$\varnothing_{v}=\varnothing_{v}{ }^{0}+S v \cdot C^{1 / 2}$

Where $\varnothing_{V}^{0}$ is the limiting apparent molar volume and $S_{v}$ a semi-empirical parameter which depends on the nature of solute, solvent as well as temperature.

Table 1(b): Densities and Apparent molar volumes of $\mathrm{KClO}_{3}$ solution in $1 \% \mathrm{KNO}_{3}$ and distilled water at different temperatures

\begin{tabular}{|c|c|c|c|c|c|c|c|c|}
\hline $\begin{array}{l}\text { Molar Conc. } \\
\text { (C) } \mathrm{mol} / \mathrm{dm}^{3}\end{array}$ & $\begin{array}{c}\mathrm{KClO}_{3} \text { in } \\
1 \% \mathrm{KCl}\end{array}$ & $\begin{array}{c}\mathrm{KClO}_{3} \text { in } 1 \% \\
\mathrm{NH}_{4} \mathrm{NO}_{3} \\
\text { Density, }(\rho)\left(\mathrm{g} . \mathrm{cm}^{-}\right.\end{array}$ & $\begin{array}{l}\mathrm{KClO}_{3} \text { in } \\
1 \% \mathrm{KNO}_{3}\end{array}$ & $\begin{array}{c}\mathrm{KClO}_{3} \text { in } 1 \% \\
\mathrm{KH}_{2} \mathrm{PO}_{4} \\
298.15 \mathrm{~K}\end{array}$ & $\begin{array}{c}\mathrm{KClO}_{3} \text { in } \\
1 \% \mathrm{KCl} \\
\text { Apparent }\end{array}$ & $\begin{array}{l}\mathrm{KClO}_{3} \text { in } 1 \% \\
\mathrm{NH}_{4} \mathrm{NO}_{3} \\
\text { lar volumes, }(\varnothing\end{array}$ & $\begin{array}{l}\mathrm{KClO}_{3} \text { in } \\
1 \% \mathrm{KNO}_{3} \\
\mathrm{~cm}^{3} \cdot \mathrm{mol}^{-1}\end{array}$ & $\begin{array}{c}\mathrm{KClO}_{3} \text { in } \\
1 \% \mathrm{KH}_{2} \mathrm{PO}_{4}\end{array}$ \\
\hline 0.0055 & 1.00376 & 1.00578 & 1.00766 & 1.01016 & 121.43 & 121.25 & 121.11 & 120.91 \\
\hline 0.0085 & 1.00462 & 1.00632 & 1.0085 & 1.01173 & 121.34 & 121.1 & 120.9 & 120.71 \\
\hline 0.0125 & 1.0055 & 1.00718 & 1.00954 & 1.01304 & 121.2 & 120.93 & 120.69 & 120.51 \\
\hline 0.0175 & 1.0066 & 1.00889 & 1.0111 & 1.01459 & 121.09 & 120.76 & 120.45 & 120.15 \\
\hline 0.0235 & 1.0079 & 1.01026 & 1.01246 & 1.01626 & 120.92 & 120.59 & 120.29 & 119.93 \\
\hline 0.0305 & 1.00945 & 1.01162 & 1.01395 & 1.01845 & 120.82 & 120.4 & 120.1 & 119.76 \\
\hline 0.0385 & 1.0111 & 1.0135 & 1.01623 & 1.02043 & 120.65 & 120.23 & 119.84 & 119.54 \\
\hline 0.0475 & 1.0129 & 1.0158 & 1.01825 & 1.02297 & 120.45 & 120.11 & 119.72 & 119.32 \\
\hline 0.0555 & 1.0144 & 1.0176 & 1.0201 & $\begin{array}{l}1.02519 \\
303.15 \mathrm{~K}\end{array}$ & 120.32 & 120 & 119.58 & 119.18 \\
\hline 0.0055 & 1.00318 & 1.00421 & 1.00615 & 1.00856 & 121.61 & 121.45 & 121.33 & 121.12 \\
\hline 0.0085 & 1.00384 & 1.00485 & 1.00692 & 1.00931 & 121.52 & 121.32 & 121.12 & 120.92 \\
\hline 0.0125 & 1.00491 & 1.00589 & 1.00807 & 1.01044 & 121.37 & 121.14 & 120.91 & 120.72 \\
\hline
\end{tabular}


RATHI., Orient. J. Chem., Vol. 37(6), 1336-1343 (2021)

\begin{tabular}{lcccccccc}
0.0175 & 1.00597 & 1.00695 & 1.00913 & 1.01159 & 121.25 & 120.96 & 120.67 & 120.36 \\
0.0235 & 1.00706 & 1.00817 & 1.01069 & 1.01356 & 121.11 & 120.78 & 120.51 & 120.14 \\
0.0305 & 1.00837 & 1.00992 & 1.01231 & 1.01535 & 121 & 120.61 & 120.32 & 119.97 \\
0.0385 & 1.00995 & 1.01157 & 1.0142 & 1.01743 & 120.8 & 120.43 & 120.06 & 119.75 \\
0.0475 & 1.0118 & 1.01324 & 1.01621 & 1.01957 & 120.65 & 120.32 & 119.94 & 119.53 \\
0.0555 & 1.0136 & 1.01516 & 1.01812 & 1.02159 & 120.51 & 120.22 & 119.8 & 119.39 \\
& & & & $308.15 \mathrm{~K}$ & & & & \\
1.00268 & 1.00381 & 1.00515 & 1.00784 & 121.76 & 121.59 & 121.45 & 121.28 & \\
0.0085 & 1.00325 & 1.00456 & 1.00639 & 1.00871 & 121.64 & 121.47 & 121.25 & 121.07 \\
0.0125 & 1.00393 & 1.00558 & 1.00708 & 1.00994 & 121.53 & 121.3 & 121.02 & 120.85 \\
0.0175 & 1.00486 & 1.00646 & 1.00853 & 1.01119 & 121.41 & 121.11 & 120.78 & 120.53 \\
0.0235 & 1.00613 & 1.00762 & 1.00989 & 1.01276 & 121.28 & 120.91 & 120.64 & 120.32 \\
0.0305 & 1.00738 & 1.00912 & 1.01161 & 1.01475 & 121.15 & 120.76 & 120.43 & 120.15 \\
0.0385 & 1.00866 & 1.01117 & 1.0134 & 1.01693 & 120.93 & 120.57 & 120.16 & 119.9 \\
0.0475 & 1.01045 & 1.01304 & 1.01581 & 1.01885 & 120.81 & 120.46 & 120.03 & 119.67 \\
0.0555 & 1.01185 & 1.01436 & 1.01752 & 1.02081 & 120.67 & 120.38 & 119.94 & 119.56 \\
& & & & $313.15 \mathrm{~K}$ & & & & 0.0055 \\
1.00196 & 1.00329 & 1.00475 & 1.00726 & & 121.92 & 121.75 & 121.64 & 121.53 \\
0.0085 & 1.00265 & 1.00406 & 1.00589 & 1.00818 & 121.82 & 121.63 & 121.47 & 121.32 \\
0.0125 & 1.00326 & 1.00518 & 1.00666 & 1.00932 & 121.69 & 121.46 & 121.24 & 121.1 \\
0.0175 & 1.00419 & 1.00597 & 1.00808 & 1.01079 & 121.57 & 121.27 & 121.01 & 120.78 \\
0.0235 & 1.00543 & 1.00712 & 1.00916 & 1.01216 & 121.43 & 121.07 & 120.83 & 120.57 \\
0.0305 & 1.00658 & 1.00872 & 1.01111 & 1.01405 & 121.29 & 120.92 & 120.66 & 120.4 \\
0.0385 & 1.00814 & 1.01017 & 1.01294 & 1.01624 & 121.16 & 120.73 & 120.41 & 120.15 \\
0.0475 & 1.00988 & 1.01224 & 1.01521 & 1.01835 & 120.97 & 120.62 & 120.26 & 119.92 \\
0.0555 & 1.01105 & 1.01376 & 1.01687 & 1.02014 & 120.87 & 120.54 & 120.19 & 119.81 \\
\hline
\end{tabular}

The apparent molar volumes of transfer at infinite dilutions $\Delta \phi_{\mathrm{v}}{ }^{0}(\mathrm{tr})$ of $\mathrm{KClO}_{3}$ obtained from the relation.

$\Delta \varnothing_{V} \mathrm{O}(\mathrm{tr})=\varnothing_{V}{ }^{0}\left(\mathrm{KClO}_{3}\right.$ in solvent system $)-\varnothing_{V}{ }^{0}\left(\mathrm{KClO}_{3}\right.$ in water)

The positive $\Delta \varnothing_{\mathrm{v}}{ }^{0}(\mathrm{tr})$ studied in the present investigation suggest that the ion-ion and ionhydrophilic group interactions are stronger than the ion hydrophobic interaction that results in an increase in volume.

The viscosity results for the aqueous solutions of $\mathrm{KClO}_{3}$ in aqueous solute systems and pure water solvent systems were plotted in accordance with Jones-Dole equation (Lomesh, Nathan, Bala , \& Thakur, 2019).

$$
\eta_{r}-1 / C^{1 / 2}=A+B C^{1 / 2}
$$

Where $\eta_{r}=\left(\eta / \eta_{0}\right)$ and $\eta, \eta_{0}$ are viscosities of the solution and solvent respectively, $\mathrm{C}$ is the molar concentration.

Table 2: Viscosities and Relative Viscosities of $\mathrm{KClO}_{3}$ solution in $1 \% \mathrm{KNO}_{3}$ and distilled water at different temperatures

\begin{tabular}{|c|c|c|c|c|c|c|c|c|}
\hline $\begin{array}{l}\text { Molar Conc. } \\
\text { (C) } \mathrm{mol} / \mathrm{dm}^{3}\end{array}$ & \multicolumn{3}{|c|}{$\begin{array}{c}\text { Viscosities }(\eta)(\mathrm{Nm}-3 . \mathrm{s}) \\
298.15 \mathrm{~K}\end{array}$} & \multicolumn{5}{|c|}{$\begin{array}{l}\text { Relative viscosities ( } \eta \mathrm{r}) \\
\text { 298.15K }\end{array}$} \\
\hline 0.0055 & 0.9296 & 0.9324 & 0.9359 & 0.9439 & 1.0358 & 1.0224 & 1.025 & 1.0239 \\
\hline 0.0085 & 0.9349 & 0.9368 & 0.9411 & 0.9491 & 1.0418 & 1.0283 & 1.0307 & 1.0289 \\
\hline 0.0125 & 0.9417 & 0.9448 & 0.9476 & 0.9556 & 1.0494 & 1.0356 & 1.0378 & 1.0335 \\
\hline 0.0175 & 0.9497 & 0.9508 & 0.9554 & 0.9624 & 1.0583 & 1.0444 & 1.0463 & 1.0474 \\
\hline 0.0235 & 0.9562 & 0.9601 & 0.9645 & 0.9715 & 1.0688 & 1.0546 & 1.0563 & 1.0579 \\
\hline 0.0305 & 0.9659 & 0.9708 & 0.9749 & 0.9799 & 1.0808 & 1.0664 & 1.0676 & 1.0699 \\
\hline 0.0385 & 0.9767 & 0.9817 & 0.9865 & 0.9915 & 1.0965 & 1.0785 & 1.0823 & 1.0856 \\
\hline .0475 & 0.9878 & 0.9938 & 0.9985 & 1.0045 & 1.1106 & 1.0924 & 1.1012 & 1.0997 \\
\hline 0.0555 & 0.9979 & $\begin{array}{c}1.0023 \\
303.15 \mathrm{~K}\end{array}$ & 1.0069 & 1.0136 & $\begin{array}{c}1.1224 \\
303.15 \mathrm{~K}\end{array}$ & $\begin{array}{l}1.1064 \\
0.0055\end{array}$ & 1.1165 & 1.1115 \\
\hline 0.8461 & 0.8504 & 0.8547 & 0.8637 & 1.0312 & 1.0261 & 1.0293 & 1.0258 & \\
\hline 0.0085 & 0.8516 & 0.8558 & 0.8604 & 0.8674 & 1.0381 & 1.0325 & 1.0361 & 1.0306 \\
\hline 0.0125 & 0.8586 & 0.8626 & 0.8674 & 0.8734 & 1.0466 & 1.0407 & 1.0446 & 1.0358 \\
\hline 0.0175 & 0.8669 & 0.8707 & 0.8759 & 0.8829 & 1.0567 & 1.0505 & 1.0548 & 1.0493 \\
\hline 0.0235 & 0.8747 & 0.8802 & 0.8858 & 0.8908 & 1.0687 & 1.0619 & 1.0668 & 1.0596 \\
\hline 0.0305 & 0.8858 & 0.8911 & 0.8972 & 0.9021 & 1.0824 & 1.075 & 1.0804 & 1.0716 \\
\hline 0.0385 & 0.8962 & 0.9021 & 0.9086 & 0.9132 & 1.1022 & 1.0914 & 1.0985 & 1.0874 \\
\hline
\end{tabular}


RATHI., Orient. J. Chem., Vol. 37(6), 1336-1343 (2021)

\begin{tabular}{|c|c|c|c|c|c|c|c|c|}
\hline 0.0475 & 0.9072 & 0.9152 & 0.9202 & 0.9262 & 1.1217 & 1.1087 & 1.1185 & 1.1016 \\
\hline \multirow[t]{2}{*}{0.0555} & 0.9187 & 0.9267 & 0.9329 & 0.9399 & 1.1418 & 1.1293 & 1.1344 & 1.1137 \\
\hline & $308.15 \mathrm{~K}$ & & & $308.15 \mathrm{~K}$ & & & & \\
\hline 0.0055 & 0.769 & 0.7749 & 0.7817 & 0.7837 & 1.0344 & 1.0379 & 1.0391 & 1.035 \\
\hline 0.0085 & 0.7752 & 0.7799 & 0.7869 & 0.7899 & 1.0434 & 1.0446 & 1.0461 & 1.0398 \\
\hline 0.0125 & 0.7824 & 0.7862 & 0.7934 & 0.7954 & 1.0548 & 1.0531 & 1.0546 & 1.045 \\
\hline 0.0175 & 0.7924 & 0.7957 & 0.8011 & 0.8057 & 1.0683 & 1.0631 & 1.0649 & 1.0585 \\
\hline 0.0235 & 0.8015 & 0.8062 & 0.8102 & 0.8162 & 1.0843 & 1.0749 & 1.0769 & 1.0688 \\
\hline 0.0305 & 0.8115 & 0.8177 & 0.8205 & 0.8255 & 1.1014 & 1.0884 & 1.0907 & 1.0808 \\
\hline 0.0385 & 0.8202 & 0.8242 & 0.8312 & 0.8362 & 1.1132 & 1.1062 & 1.1048 & 1.0966 \\
\hline 0.0475 & 0.8322 & 0.8382 & 0.8422 & 0.8482 & 1.1265 & 1.1156 & 1.1123 & 1.1108 \\
\hline \multirow[t]{2}{*}{0.0555} & 0.8457 & 0.8517 & 0.8547 & 0.8577 & 1.1398 & 1.1258 & 1.1221 & 1.1229 \\
\hline & $313.15 \mathrm{~K}$ & & & & $313.15 \mathrm{~K}$ & & & 0.0055 \\
\hline 0.6832 & 0.6939 & 0.7056 & 0.7147 & 1.0293 & 1.0346 & 1.0398 & 1.037 & \\
\hline 0.0085 & 0.6901 & 0.7006 & 0.7125 & 0.7236 & 1.0396 & 1.0447 & 1.0498 & 1.0418 \\
\hline 0.0125 & 0.701 & 0.7091 & 0.7211 & 0.7323 & 1.056 & 1.0573 & 1.0624 & 1.0473 \\
\hline 0.0175 & 0.7127 & 0.7192 & 0.7323 & 0.7437 & 1.0735 & 1.0724 & 1.0776 & 1.0605 \\
\hline 0.0235 & 0.7265 & 0.7356 & 0.7442 & 0.7562 & 1.0939 & 1.0901 & 1.0952 & 1.0708 \\
\hline 0.0305 & 0.741 & 0.7496 & 0.7629 & 0.7735 & 1.1175 & 1.1102 & 1.1154 & 1.0828 \\
\hline 0.0385 & 0.7613 & 0.7693 & 0.7814 & 0.7932 & 1.1256 & 1.1234 & 1.1278 & 1.0986 \\
\hline 0.0475 & 0.7798 & 0.7928 & 0.8022 & 0.8139 & 1.1387 & 1.1343 & 1.1366 & 1.1128 \\
\hline 0.0555 & 0.7974 & 0.8094 & 0.8178 & 0.8313 & 1.1475 & 1.1433 & 1.1458 & 1.1249 \\
\hline
\end{tabular}

The B-coefficients were obtained from the $\eta_{r}^{2}=M+K C^{2}$

linear plots using the least-square fitting method.

The A-coefficient reflects solute-solute interaction (Pérez-Durán, 2019) and the B-coefficient reflect the solute-solvent interactions. Since in general, $A / B$ $<<1$, the Jones -Dole equation reduces to,

$\eta_{r}=I+\beta \cdot C$

The relative viscosity data of these solutions have also been fitted in Moulik equation,
The density data of these solutions have also been fitted in Root's equation,

$\left(d-d_{0}\right) / C=R-S C^{1 / 2}$

where $\mathrm{R}$ and $\mathrm{S}$ are constants.

The diffusion controlled reaction rate constant $k_{d}$ can be evaluated by using the viscosity data as (Rathi \& Nikumbh, 2019).

Table 3: Different parameters of $\mathrm{KClO}_{3}$ in water and $1 \% \mathrm{KCl}, \mathrm{KNO}_{3}, \mathrm{NH}_{4} \mathrm{NO}_{3}$ and $\mathrm{KH}_{2} \mathrm{PO}_{4}$ solution

\begin{tabular}{|c|c|c|c|c|c|c|c|c|c|c|}
\hline Temp & $\begin{array}{c}\text { ssons Paramet } \\
\varnothing_{V_{0}} \\
\mathrm{~cm}^{3} \cdot \mathrm{mol}^{-1} \mathrm{~L}^{1 / 2}\end{array}$ & $\begin{array}{l}\text { rs } \underset{\mathrm{S}_{\mathrm{v}}}{\text { Jor }} \\
\mathrm{cm}^{3} \cdot \mathrm{mol}^{-1}\end{array}$ & $\begin{array}{c}\text { Dole's Param } \\
\text { A/ } \\
\mathrm{dm}^{3 / 2} \mathrm{~mol}^{-1 / 2}\end{array}$ & $\begin{array}{l}\text { B/ } \\
\mathrm{dm}^{3} \cdot \mathrm{mol}^{-1}\end{array}$ & $\begin{array}{c}\text { Moulik Param } \\
\text { K }\end{array}$ & ${ }_{M}{ }^{F}$ & $\begin{array}{c}\text { Roots Parar } \\
\text { R }\end{array}$ & $\mathrm{S}$ & $\begin{array}{c}\beta \text { ' values } \\
\beta\end{array}$ & $\varnothing_{V}^{\stackrel{\Delta}{0}}(\mathrm{tr})$ \\
\hline \multicolumn{11}{|c|}{$\mathrm{KClO}_{3}$ in Water } \\
\hline 298.15K & 121.3 & -10.21 & -0.01 & 0.32 & 12.61 & 1 & 0.24 & 0.29 & 0.28 & 0 \\
\hline $303.15 \mathrm{~K}$ & 121.2 & -8.85 & -0.04 & 1.09 & 43.44 & 1.01 & 0.26 & 0.3 & 0.95 & 0 \\
\hline $308.15 \mathrm{~K}$ & 121.9 & -10.92 & -0.05 & 2.01 & 84.38 & 1.03 & 0.24 & 0.27 & 1.81 & 0 \\
\hline $313.15 \mathrm{~K}$ & 121.3 & -10.19 & -0.03 & 1.32 & 55.23 & 1.02 & 0.26 & 0.3 & 1.2 & 0 \\
\hline \multicolumn{11}{|c|}{$\mathrm{KClO}_{3}$ in $1 \% \mathrm{KCl}$} \\
\hline $298.15 K$ & 121.7 & -4.05 & 0.03 & 0.35 & 40.18 & 1.04 & 0.52 & -1.39 & 0.87 & 0.4 \\
\hline $303.15 \mathrm{~K}$ & 121.9 & -4.03 & 0.05 & 0.93 & 55.7 & 1.03 & 0.53 & -1.5 & 1.22 & 0.7 \\
\hline $308.15 \mathrm{~K}$ & 122.1 & -3.89 & -0.03 & 1.58 & 74.91 & 1.03 & 0.54 & -1.5 & 1.61 & 0.2 \\
\hline $313.15 K$ & 122.2 & -3.16 & 0.03 & 1.79 & 99.02 & 1.04 & 0.55 & -1.6 & 2.1 & 0.9 \\
\hline \multicolumn{11}{|c|}{$\mathrm{KClO}_{3}$ in $1 \% \mathrm{NH}_{4} \mathrm{NO}_{3}$} \\
\hline 298.15K & 121.8 & -7.71 & 0 & 1.14 & 52.04 & 1.02 & 1.45 & -5.53 & 1.13 & 0.5 \\
\hline $303.15 K$ & 122.1 & -7.81 & 0.02 & 1.21 & 58.8 & 1.02 & 1.47 & -5.7 & 1.27 & 0.9 \\
\hline $308.15 \mathrm{~K}$ & 122.2 & -7.56 & 0.01 & 1.52 & 72.89 & 1.03 & 1.28 & -4.75 & 1.57 & 0.3 \\
\hline $313.15 K$ & 122.4 & -7.77 & 0.01 & 2.06 & 97.91 & 1.04 & 1.53 & -6.03 & 2.08 & 1.1 \\
\hline \multicolumn{11}{|c|}{$\mathrm{KClO}_{3}$ in $1 \% \mathrm{KNO}_{3}$} \\
\hline $298.15 K$ & 121.9 & -10.63 & -0.03 & 1.57 & 57.27 & 1.03 & 1.45 & -5.17 & 1.47 & 0.6 \\
\hline $303.15 K$ & 122.1 & -10.53 & 0.1 & 0.86 & 68.16 & 1.02 & 1.46 & -5.21 & 1.24 & 0.9 \\
\hline $308.15 \mathrm{~K}$ & 122.3 & -10.69 & 0.06 & 1.17 & 64 & 1.03 & 1.5 & -5.5 & 1.38 & 0.4 \\
\hline $313.15 \mathrm{~K}$ & 122.6 & -11.18 & -0.02 & 1.94 & 86.68 & 1.03 & 1.39 & -4.86 & 1.86 & 1.3 \\
\hline \multicolumn{11}{|c|}{$\mathrm{KClO}_{3}$ in $1 \% \mathrm{KH}_{2} \mathrm{PO}_{4}$} \\
\hline $298.15 \mathrm{~K}$ & 123.1 & -10.25 & 0.02 & 1.57 & 56.32 & 1.03 & 1.46 & -5.27 & 1.27 & 1.8 \\
\hline $303.15 \mathrm{~K}$ & 123.3 & -9.73 & 0.15 & 0.86 & 66.17 & 1.02 & 1.45 & -5.23 & 1.23 & 2.1 \\
\hline $308.15 \mathrm{~K}$ & 123.5 & -10.55 & 0.03 & 1.17 & 71.24 & 1.03 & 1.53 & -5.51 & 1.27 & 1.6 \\
\hline $313.15 \mathrm{~K}$ & 123.7 & -10.7 & 0.02 & 1.94 & 89.35 & 1.03 & 1.29 & -4.76 & 1.46 & 2.4 \\
\hline
\end{tabular}


$\mathrm{k}_{\mathrm{d}}=\frac{8 R T}{3 \eta}$

The evaluated values are used to predict

Table 4: Diffusion reaction rate constant $\mathrm{k}_{\mathrm{d}}\left(\mathrm{L} \mathrm{mol}^{-1} \mathrm{~s}^{-1}\right)$ values of $\mathrm{KCIO}_{3}$ water and $1 \% \mathrm{KCl}, \mathrm{KNO}_{3}, \mathrm{NH}_{4} \mathrm{NO}_{3}$ and $\mathrm{KH}_{2} \mathrm{PO}_{4}$ solution

\begin{tabular}{|c|c|c|c|c|c|c|c|c|c|c|}
\hline $\begin{array}{l}\text { Molar Conc. } \\
\mathrm{mol} / \mathrm{dm}^{3}\end{array}$ & $\begin{array}{l}\mathrm{KClO}_{3} \\
\text { in Water }\end{array}$ & $\begin{array}{c}\mathrm{KClO}_{3} \text { in } \\
1 \% \mathrm{KCl}\end{array}$ & $\begin{array}{c}\mathrm{KClO}^{3} \text { in } \\
1 \% \mathrm{NH}_{4} \mathrm{NO}_{3}\end{array}$ & $\begin{array}{l}\mathrm{KClO}_{3} \text { in } \\
1 \% \mathrm{KNO}_{3}\end{array}$ & $\begin{array}{c}\mathrm{KClO}_{3} 1 \% \\
\mathrm{KH}_{2} \mathrm{PO}_{4}\end{array}$ & $\begin{array}{l}\mathrm{KClO}_{3} \\
\text { in Water }\end{array}$ & $\begin{array}{c}\mathrm{KClO}_{3} \text { in } \\
1 \% \mathrm{KCl}\end{array}$ & $\begin{array}{c}\mathrm{KClO}_{3} \text { in } \\
1 \% \mathrm{NH}_{4} \mathrm{NO}_{3}\end{array}$ & $\begin{array}{l}\mathrm{KClO}_{3} \text { in } \\
1 \% \mathrm{KNO}_{3}\end{array}$ & $\begin{array}{c}\mathrm{KClO}_{3} 1 \% \\
\mathrm{KH}_{2} \mathrm{PO}_{4}\end{array}$ \\
\hline & & & $298.15 K$ & & & & & $298.15 \mathrm{~K}$ & & \\
\hline 0.0055 & 7.38 & 7.23 & 7.21 & 7.18 & 7.16 & 9.35 & 9.12 & 9.05 & 8.97 & 8.21 \\
\hline 0.0085 & 7.37 & 7.21 & 7.18 & 7.14 & 7.13 & 9.28 & 9.06 & 9.01 & 8.92 & 8.14 \\
\hline 0.0125 & 7.36 & 7.18 & 7.14 & 7.09 & 7.06 & 9.2 & 8.99 & 8.93 & 8.86 & 7.92 \\
\hline 0.0175 & 7.35 & 7.14 & 7.09 & 7.03 & 7.04 & 9.1 & 8.91 & 8.85 & 8.79 & 7.84 \\
\hline 0.0235 & 7.34 & 7.11 & 7.03 & 6.96 & 6.94 & 8.99 & 8.81 & 8.75 & 8.71 & 7.74 \\
\hline 0.0305 & 7.32 & 7.09 & 6.97 & 6.88 & 6.86 & 8.87 & 8.7 & 8.65 & 8.62 & 7.65 \\
\hline 0.0385 & 7.31 & 7.06 & 6.83 & 6.74 & 6.73 & 7.91 & 7.83 & 8.56 & 8.38 & 7.56 \\
\hline 0.0475 & 7.3 & 7.04 & 6.76 & 6.56 & 6.56 & 7.87 & 7.71 & 8.34 & 8.17 & 7.38 \\
\hline \multirow[t]{2}{*}{0.0555} & 7.29 & 7.01 & 6.45 & 6.43 & 6.4 & 7.68 & 7.64 & 8.11 & 8.01 & 7.29 \\
\hline & & & $303.15 \mathrm{~K}$ & & & & & $303.15 \mathrm{~K}$ & & \\
\hline 0.0055 & 8.27 & 8.09 & 8.03 & 7.98 & 8.19 & 10.55 & 10.28 & 10.12 & 10.12 & 10.23 \\
\hline 0.0085 & 8.24 & 8.05 & 7.99 & 7.94 & 8.04 & 10.5 & 10.19 & 10.05 & 10.05 & 10.14 \\
\hline 0.0125 & 8.2 & 8.01 & 7.94 & 7.9 & 7.89 & 10.44 & 10.09 & 9.96 & 9.96 & 9.95 \\
\hline 0.0175 & 8.15 & 7.95 & 7.88 & 7.84 & 7.74 & 10.37 & 9.97 & 9.85 & 9.85 & 9.86 \\
\hline 0.0235 & 8.1 & 7.88 & 7.82 & 7.77 & 7.63 & 10.28 & 9.83 & 9.73 & 9.73 & 9.72 \\
\hline 0.0305 & 8.04 & 7.81 & 7.74 & 7.7 & 7.55 & 10.19 & 9.68 & 9.59 & 9.59 & 9.68 \\
\hline 0.0385 & 7.89 & 7.23 & 7.66 & 7.68 & 7.46 & 10 & 9.46 & 9.33 & 9.47 & 9.57 \\
\hline 0.0475 & 7.67 & 7.11 & 7.54 & 7.43 & 7.31 & 9.89 & 9.32 & 9.24 & 9.33 & 9.45 \\
\hline 0.0555 & 7.43 & 6.89 & 7.23 & 7.1 & 7.2 & 9.76 & 9.21 & 9.12 & 9.28 & 9.29 \\
\hline
\end{tabular}

\section{RESULT AND DISCUSSION}

The values of densities and viscosities of $\mathrm{KClO}_{3}$ solutions decrease with increase in temperature and increases with increase in concentration. The apparent molar volume values of $\varnothing_{V}$ decrease with concentration while the relative viscosities are found to increase with concentrations. The $\varnothing_{\mathrm{v}}{ }^{0}$ values of $\mathrm{KClO}_{3}$ under investigation in all solvent systems are large and positive. The $S_{v}$ is negative. These results indicate that there is presence of strong solute-solute interactions. (Shekaari, 2019) The Diffusion reaction rate constant $\left(k_{d}\right)$ values are positive and decrease with increase in temperature as well as concentration. Positive $\mathrm{Kd}$ values reveals that the solvolysis of $\mathrm{KClO}_{3}$ in aqueous electrolyte studied is diffusion controlled rather than activated controlled process.

\section{CONCLUSION}

The increase in densities and viscosities with concentration may be due to strengthening of solute-solvent interactions. (Gupta, 2019). All the investigated parameters has been revealed that there exist strong solute-solvent interaction.
This molecular interaction data help to reveal the toxicity of chlorate. When chlorate is added in the soil or injected in the stem of Longan plants, it creates a strong solute-solvent interaction with the other aqueous ionic solids which are already present in the soil or plant tissues. Chlorate acts as a structure maker and form a tight solvation layer as well as high compactness around the solute. Over-application of flower-inducing chlorate failed to induce flower but caused toxic effects such as leaf chlorosis and drop in longan. The solvolysis of $\mathrm{KClO}_{3}$ in water and $\mathrm{KNO}_{3}$ or other nitrate and phosphate solutions at different temperatures is diffusion controlled process. The co-sphere of solvent significantly suppressed nitrate reducing activity in the leaves. This suggested that chlorate not only inhibited photosynthesis due to stomata closure but also due to breakdown of photosynthetic apparatus in chloroplasts. (Sritontip C. Y., 1999) The higher values of densities of $\mathrm{KClO}_{3}$ in $\mathrm{KCl}$, $\mathrm{KNO}_{3}, \mathrm{NH}_{4} \mathrm{NO}_{3}$ and $\mathrm{KH}_{2} \mathrm{PO}_{4}$ are due to the relative salvation, corresponding relative volumes of system and molar mass of the salts. Strong molecular interactions induces chlorate stress (LU Jiemei, 2017) due to occurrence of osmotic adjustment which results in massive accumulation of soluble 
amino acids and sugars. Excessive use of chlorate as a bleaching agent toxifies the water (Lubbers, Chauhan, \& Bianchine, 1981), soil and disturbs the environment's equilibrium. (Kanitz, 1996). Reducing the usage of chlorate, used in the production of colours, safety matches, and explosives for tanning and finishing leather, is important in order to conserve and save the environment.

\section{ACKNOWLEDGEMENT}

I would like to sincerely acknowledge the guidance and help provided by Dr. Arun B. Nikumbh for compilation of this research report.

\section{REFERENCES}

1. Retrieved from https://www.businesswire. com/portal/site/home/my-business-wire/: https://www.businesswire.com/portal/site/ home/my-business-wire/., 2020.

2. Bahadur, I. M. Intermolecular interactions between methanol and some sulphonamide drugs in aqueous medium using thermodynamics approach. Journal of Molecular Liquids., 2019, 283, 451-461. doi:10.1016/j.molliq.2019.03.065.

3. Caro, R. H. Molar Volumes and Heat Capacities of Aqueous Solutions of $\mathrm{Mg}\left(\mathrm{ClO}_{4}\right)$ 2. Journal of Chemical \& Engineering Data., 2020, 65(7), 3735-3743.

4. Cui, W., Hou, H., Chen, J., Guo, Y., Meng, L., \& Deng, T. Apparent molar volumes of sodium arsenate aqueous solution from 283.15 K to $363.15 \mathrm{~K}$ at ambient pressure: an experimental and thermodynamic modeling study. Pure and Applied Chemistry., 2020, 92, 1673-1682. doi:10.1515/pac-2019-1102.

5. Daniel, F. Comparative subchronic toxicity studies of three disinfectants. Journal of the American Water Works Association., 1990, 82, 61-69.

6. Ganjare, P. Prediction of molecular interactions in liquids from density., Vidyabharati International Interdisciplinary Research Journal., 2020, 479-499.

7. Gupta, J. \&. Molecular interactions of gentamicin sulphate in aqueous-Iasparagine/l-glutamine solutions at different temperatures: Volumetric, acoustic and viscometric properties. Journal of Molecular Liquids., 2019, 111547.

8. Hegele, M. N. Effect of leaf age on the response of flower induction and related hormonal changes in longan trees after $\mathrm{KClO}_{3}$ treatmen. In IX International Symposium on Plant Bioregulators in Fruit Production., 2001, 41-49.

9. Kanitz, s. Association between drinking water disinfection and somatic parameters at birth.
Environmental Health Perspectives., 1996, 104(5), 516-520.

10. Kharat, S. J. Density and viscosity studies of binary mixtures of aniline+ benzene and ternary mixtures of (aniline+ benzene+ $\mathrm{N}$, $\mathrm{N}$-dimethylformamide) at 298.15, 303.15, 308.15 , and $313.15 \mathrm{~K}$. Journal of molecular liquids., 2007, 131, 81-86.

11. Langore, K., Nikumbh, A., Patil, S., \& Gaikwad, S. Apparent Molar Volumes And Viscosity B Coefficients Of Chlorothalonil Pesticide In Binary Mixture DMF And DMSO At Different Temperatures. International Journal of Scientific Research., 2019, 8, 1489-1498., doi:https://doi.org/10.7897/22308407.110661.

12. Lomesh, S.; Nathan, V.; Bala, M.; \& Thakur, $P$. Volumetric and acoustic methods for investigating molecular interactions of antibiotic drug doxycycline hyclate in water and in aqueous solution of sodium chloride and potassium chloride at different temperatures (293.15-313.15) K. Journal of Molecular Liquids., 2019, 284, 241-251. doi:10.1016/j.molliq.2019.04.006.

13. LU Jiemei, Y. R. Stress Effects of Chlorate on Longan (Dimocarpus longan Lour.). Horticultural Plant Journal., 2017, 3(6), 237-246.

14. Lubbers, J.; Chauhan, S., \& Bianchine, J. Controlled clinical evaluations of chlorine dioxide,chlorite and chlorate in man. Fundamental and Applied Toxicology., 1981, 1, 334-338.

15. Manochai, P. J. Seasonal effect of potassium chlorate on flowering and yield of longan (Dimocarpus longan Lour.). III International Symposium on Longan, Lychee, and other Fruit Trees in Sapindaceae Family., 2008, 863, 363-366.

16. Manochai, S. W.-a. Effect of potassium chlorate for inducting off longan cvs. 0Do1 and Sri-Chompu. Seminar on Using Plant Hormone for Off-season Production in Fruit Trees. Chantaburi, Thailand., 1999, 1-8. 
17. Moore, G., \& Calabrese, E. Toxicological effects of chlorite in the mouse. Environmental Health Perspectives., 1982, 46, 31-37.

18. Nahar, N. C. Effects of $\mathrm{KClO}_{3}, \mathrm{KNO}_{3}$ and urea on the flowering and fruiting of mango and longan. J of Agro Environ., 2010, 4(1), 31-34.

19. Nain, A. K. Physicochemical study of solutesolute and solute-solvent interactions of I-histidine in water+ sucrose solutions at different temperatures. Journal of Molecular Liquids. Journal of Molecular Liquids., 2012, 165, 154-160.

20. Nanda, B.; Nanda, B., \& Dalai, B. Effect of the Temperature and Solvents on the Solvolysis of Barium Bromide in Aqueous-Organic Solutions: Volumetric and Viscometric Study. Biointerface Research in Applied Chemistry., 2021, 12(1), 339-350.

21. Nanda, B.; Nanda, B., \& Mohanty, P. Effect of concentration on thermo acoustic and nonlinearity parameters $(B / A)$ of barium chloride solutions in glycol-water mixtures at 303.15K. Journal of Molecular liquids., 2012 , 171, 50-53. doi:10.1016/j.molliq.2012.03.011.

22. Pérez-Durán, G. \&.-S. Densities and Viscosities for Aqueous Solutions of Sodium Chlorate and Potassium Chlorate+ Methanol from (288.15 to 318.15$) \mathrm{K}$ at $0.1 \mathrm{MPa}$. Journal of Chemical \& Engineering Data., 2019. 64(5), 1999-2010.

23. Rathi, M., \& Nikumbh, A. Interactions of
$\mathrm{KIO}_{3}$ in aqueous $0.1 \%$. Journal of Emerging Technologies and Innovative Research., 2019, 6(1), 167-175.

24. Shahrajabian, M. H. Modern pharmacological actions of longan fruits and their usages in traditional herbal remedies. Journal of Medicinal Plants Studies., 2019, 7(4), 179-185.

25. Shekaari, H. A. Understanding solvation behavior of glucose in aqueous solutions of some deep eutectic solvents by thermodynamic approach. Journal of Molecular Liquids., 2019, 289, 111000.

26. Snyder SA, P. R. Perchlorate and chlorate in dietary supplements and flavor enhancing ingredients. Anal ChimActa., 2006, 567(1), 26-32.

27. Sritontip, C. Y. Effect of potassium chlorate on flowering in longan cv. In. Seminar on Using Plant Hormone for Off-Season Fruits Crop., Chantaburi, Thailand., 1999, 21-28.

28. Sritontip, C. Y. Effect of potassium chlorate, potassium nitrate, sodium hypochlorite and thiourea on off-season flowering and photosynthesis of'Do'longan. II International Symposium on Lychee, Longan, Rambutan and other Sapindaceae Plants., 2003, 291-296.

29. Viriya-alongkorn, W. V. Preliminary study on stem injection of potassium chlorate on flowering and fruit setting of longan (Dimocarpus longan. In. Seminar on Using Plant Hormone for Offseason Production in Fruit Trees, Chantaburi, Thailand., 1999, 15-20. 